\title{
Reducing Logistic Vehicle Kilometers in a City Area based on Network Changes
}

\author{
Pieter Vansteenwegen \\ KU Leuven, Leuven Mobility Research Center - CIB \\ pieter.vansteenwegen@kuleuven.be
}

\author{
Corrinne Luteyn \\ KU Leuven, Leuven Mobility Research Center - CIB \\ corrinne.luteynakuleuven. be
}

\begin{abstract}
In this research, we look for the possible reduction in vehicle kilometers that can be obtained when a cooperation of delivery companies has the ability to suggest network improvements to the local government. Different network changes are considered in our research: re-opening existing roads for the vehicles of the cooperation, widening roads in the network or converting existing roads into a one-way road with a higher speed. To find the best set of improvements given a fixed budget in a realistic road network, an Adaptive Large Neighborhood Search (ALNS) is proposed. Both the destroy and repair methods in this ALNS are unique for this problem. In order to get an indication of the possible reduction in vehicle kilometers and to test the performance of the heuristic, experiments on a set of 16 benchmark instances are executed. These benchmark instances are generated from a realistic city road network. Based on these experimental results, we can conclude that a set of 1 up to 4 network improvements can lead to a reduction in vehicle kilometers of on average around $2.4 \%$ over these 16 benchmark instances, while implementing a set of 2 up to 9 improvements can lead to a reduction of on average around $3.3 \%$ over this set of benchmark instances.
\end{abstract}

\section{INTRODUCTION}

In the last decades, the number of vehicles delivering products in city areas has increased enormously. Not only the shops and companies in the area require a delivery of their ordered goods, but also the residents of the city which ordered their new products online. Recent research [2] showed that horizontal cooperation of these deliveries will reduce both the city congestion well as the $\mathrm{CO}_{2}$ emissions in the city area. This horizontal cooperation has also positive effects for the involved companies, since their unit costs will decrease and their service will improve. The local government, as policy maker, can play a large role in stimulating this cooperation [7].

Since combining deliveries of different companies has positive effects on both the accessibility of the city area and the environment, actually, more and more local governments stimulate and facilitate the cooperation between different delivering companies. This can be done in different ways, for example by financial support or by adapting the accessibility of certain roads in the city area. This could save vehicle kilometers for the delivery companies and $\mathrm{CO}_{2}$ emissions for the city area [7]. In this research, we focus on the savings that can be obtained when this cooperation of companies has the ability to discuss road network changes with the local government. We assume that there is a fixed budget available to adjust the network.
Within this given budget, the cooperation can suggest a set of network changes to the local government.

We consider three different improvements of the road network. The first improvement is (re-)opening a road for the vehicles of the cooperation. This road might be for example a pedestrian street which will be (re-)opened for delivery trucks (during some hours of the day). Another possibility is to widen a road, while the third possibility is the transformation of a road into a one-way street with a higher speed. All three changes have an effect on the travel time (and distance) between two locations. This new travel time will also have an influence on the travel time between other location pairs if their shortest connection contains this new or widened road. When a two-way road is converted into a one-way street, this also has a negative effect on the distance for the traffic in the opposite direction. Consequently, in this case, the shortest connection between certain location pairs could be negatively impacted, since a part of the current connection is no longer available.

The main goal of this research is to develop a procedure to find the best set of improvements in a road network within the given budget, in order to minimize the total number of kilometers of the vehicles of the cooperation. Since the set of customers that require a visit varies from day to day, this best set of improvements is determined over a large set of days. We will present an Adaptive Large Neighborhood Search (ALNS) to solve the problem. In order to obtain an indication of the possible savings and to test the performance of our solution approach, a set of 16 benchmark instances is generated from a realistic city road network. This set of benchmark instances is considered with two different available budgets.

\section{PROBLEM DESCRIPTION}

The construction of routes for the vehicles of the cooperation of transport companies can be transformed into a new variant of the Vehicle Routing Problem (VRP). Two of the main assumptions in the normal VRP are that the considered network is complete and consequently, that all nodes in the network are customer locations. However, in a realistic city road network, a direct path is not always available between each pair of nodes, or in other words, the network is incomplete. This means that nodes can be passed by multiple times, which is not allowed in the original VRP. Furthermore, in a city area, there are many known customers, however, 
on a single day, only a part of them has a positive demand and should be served by a vehicle. Therefore, we introduce the Steiner Vehicle Routing Problem (SVRP), in which the considered network is incomplete and nodes might be visited multiple times. Furthermore, in the SVRP, only a selection of the known customers has a positive demand and requires a visit of a vehicle.

The SVRP with multiple vehicles is not yet introduced in literature. However, the variant with only one vehicle, the Steiner Traveling Salesman Problem (STSP), is studied several times. The STSP is introduced by Fleischmann in [5], which has presented a MIP formulation for the problem. In [9], different compact formulations of the STSP are discussed in order to reduce the number of both the variables and the constraints, compared to the traditional formulation.

Note that in the SVRP and also in the STSP, only the routing of the customers is considered, which means that the determination of the best set of improvements is not included. In order to determine the best set of improvements of a road network given a fixed budget, we introduce the Steiner Vehicle Routing Problem with a Budget Constraint (SVRBC). Since the group of known customers with a positive demand varies from day to day, the best set of network improvements is determined over a set of days.

In most routing problems presented in literature, it is assumed that the considered network is fixed and known in advance. However, in other research fields, changing networks are studied, such as sensor networks (see e.g. [1]) and ad-hoc or wireless networks (see e.g. [4]). In these studies, however, the routes need to be reoptimized when a given change in the network occurs. In our research, we are going one step further, since deciding on the possible changes of the network is a part of the optimization process. Therefore, our problem is more complex than the problems with changing networks in the state of the art.

\section{AdAPtive LARGE NeIGHBORHOOd SEARCH}

In this section, we present our solution approach to determine the best combination of improvements, given a budget constraint. This heuristic implements the Adaptive Large Neighborhood Search (ALNS) framework of Ropke et al., presented in [10]. The main structure of our heuristic is a Simulated Annealing (SA) algorithm, where the next solution is every time generated by a large neighborhood search. This large neighborhood consists of a set of destroy and a set of repair methods. These destroy and repair methods are unique for this problem, since, in this case, the best combination of improvements should be determined instead of the best routes for the vehicles in normal routing problems. Every time, after a fixed number of iterations, the weights of the destroy and repair methods are adjusted, based on their performance during these iterations, and the ALNS continues with these new weights. For every newly generated solution, i.e. a set of network improvements, the required vehicle kilometers on this adjusted network are estimated by a Variable Neighborhood Search (VNS). Therefore, this VNS is used to solve in a fast

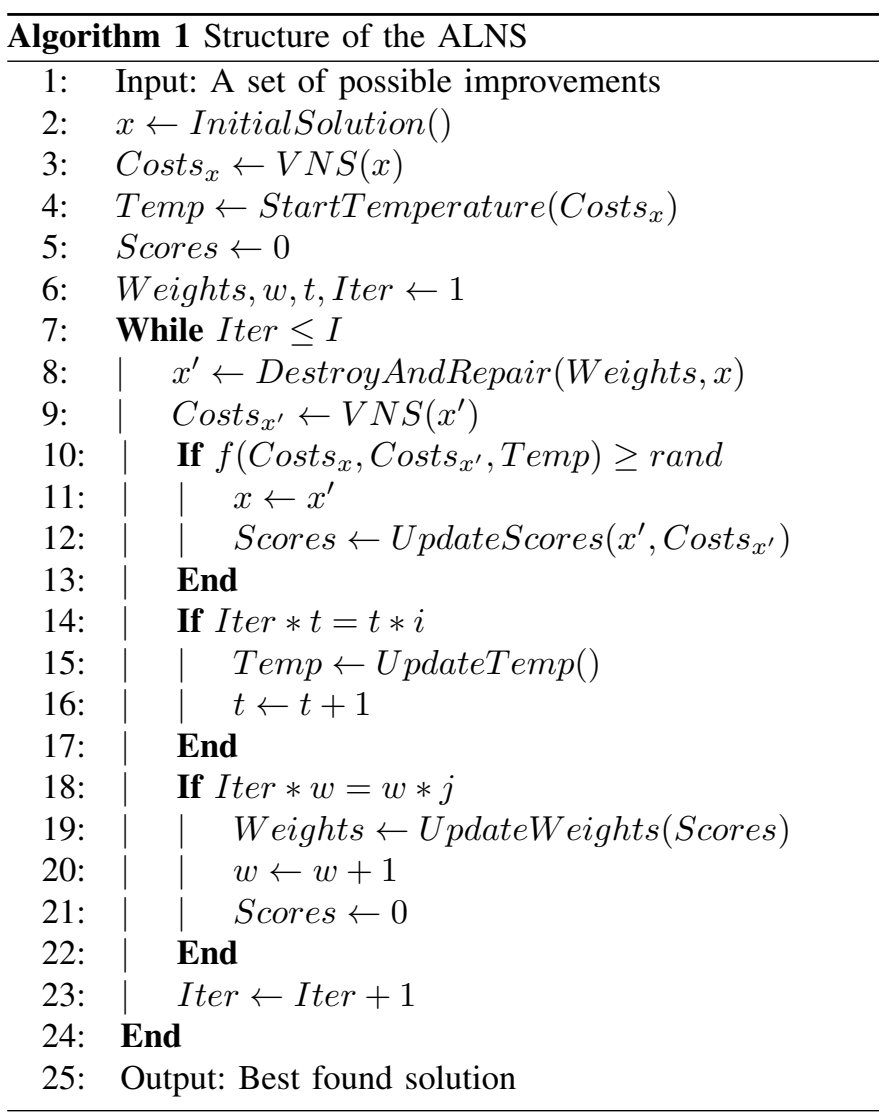

way the SVRP for each day of the set of days considered. At the end of the ALNS, the set of network improvements resulting in the least estimated vehicle kilometers is returned as best solution. The structure of our ALNS is displayed in Algorithm 1. Both the ALNS framework and a VNS heuristic are frequently used to solve complex transportation problems in recent research (see e.g. [3], [11], [12] and [13]). In the remaining part of this section, the main components of the ALNS are discussed in more detail.

\section{A. Initial Solution}

The initial set of improvements to start our ALNS is randomly generated. The first improvement of this initial set is randomly selected from all possible improvements, while the next improvements are chosen, one by one, randomly from all possible improvements which fit in the remaining budget.

\section{B. Simulated Annealing (SA)}

In the structure of SA, better solutions are always accepted and worse solutions are sometimes accepted. Whether a worse solution will be accepted, is dependent of the SA 'temperature'. In our ALNS, the start temperature is determined based on the estimated amount of vehicle kilometers in the network adjusted by the initial solution. The probability that a new solution with a fixed percentage $p$ more vehicle kilometers than the initial solution is accepted, is set to be equal to 0.5. After a fixed number $i$ of iterations, this temperature is multiplied by $\alpha$ with $(0<\alpha<1)$. Since the temperature decreases, the 
probability of accepting worse solutions decreases. Therefore, at the end of the heuristic, the solution converges to a (local) optimum.

\section{Destroy Methods}

In our ALNS, four destroy methods are used. In each method, a fixed percentage $d$ of the improvements in the solution is removed. The first method is the random destroyment. In this method, the removed improvements are chosen randomly, while in the second one, the most expensive improvements in the solution are removed. In the third method, the solution is destroyed by removing the improvements which are in the solution for the largest number of iterations. For the last destroy method, the impact of each improvement in the solution should be estimated. This is done by analyzing the routes in the network adjusted by the improvements of the current solution. This analysis is based on the estimation of the benefit of each possible improvement, which is discused in III-D1 and III-D2. However, in this case, the realized benefit by each improvement of the current solution is determined instead of estimating the benefit for all possible improvements. The improvements with the smallest impact, which is the smallest realized benefit, are removed.

\section{Repair Methods}

Also four repair methods are used in our ALNS. In each of these methods, improvements of the list of all possible improvements are inserted in the destroyed solution until none of the possible improvements can be added without exceeding the fixed budget. In the first repair method, improvements are randomly added to the solution, while in the second one, the cheapest possible improvements are inserted in the solution. In the third method, the inserted improvements are randomly chosen from a list of possible improvements which were never used in previous solutions. For the last repair method, the benefit of all possible improvements should be estimated. This is done by analyzing the routes for the vehicles in the original road network. The most beneficial improvements are added then to the solution. This estimation of the benefit is different for each type of improvement:

1) The estimation of the benefit of adding an extra edge: To estimate the benefit of the addition of an extra edge, the number of appearances of all "couples" of locations in all routes for all days in the original network are determined. A pair of two locations in a route is considered as a "couple" if they are not visited consecutively and the locations which are visited in between these two locations are all different from one another and from the locations in the pair. Based on the number of appearances of a couple and the difference in travel distance between traveling on the shortest path of existing roads and traveling on a direct connection between the two locations of the couple, an estimation of the reduction of the travel distance by adding the road is determined.

2) The estimation of the benefit of converting an edge or widening an arc: Both the estimation of the benefit of the widening of an existing arc and of the conversion of an existing edge are based on the number of usages of the existing roads in the constructed routes. To estimate the benefit of each candidate adjustment, the number of usages of that candidate adjustment is multiplied by the reduction in travel time due to the increase of capacity on that road. In case of conversion, an estimation of the extra travel distance in the opposite direction is determined, which is subtracted from the calculated benefit.

\section{E. Adaptive Weights}

All destroy and repair methods start with the same weight, which means that the probability of each method to be chosen is equal. During the ALNS, these weights will be adjusted according to the performance of the corresponding method. First of all, the score $\pi_{i}$ of each destroy and repair method should be determined over a fixed number of iterations $j$. At the beginning of this number of iterations, the score of each method is set to zero. If a combination of destroy and repair methods is able to generate a new solution with lower estimated vehicle kilometers than the best found solution up to this far, the score of both the destroy and the repair method is increased by a fixed value $\sigma_{1}$. If the number of vehicle kilometers of the generated solution is lower than the vehicle kilometers of the current solution, the score of both methods is increased by $\sigma_{2}$. In case that the newly generated solution is accepted due to the simulated annealing structure, but the estimated vehicle kilometers of this new solution are larger than the current number of vehicle kilometers, the score of the destroy and repair method is increased by $\sigma_{3}$. After the fixed number of iterations, the weights of all methods are adjusted. For each method $i$, the new weight $w_{n}$ is calculate by

$$
w_{n}=w_{o}(1-r)+r \frac{\pi_{i}}{\theta_{i}}
$$

with $w_{o}$ is the old weight of the method and $\theta_{i}$ the number of usage during the fixed number of iterations. Parameter $r$ is a fractional parameter between zero and one $(0 \leq r \leq 1)$ which indicates the reaction factor of the weights to the performance of the methods. If $r$ is equal to zero, the weights are not adjusted to the performances, while in case $r$ is equal to one, the new weight is only depended of the performance of the method during the last fixed number of iterations.

\section{F. Variable Neighborhood Search}

In our ALNS, the evaluation of a certain solution, i.e. the estimation of the vehicle kilometers for a given set of network improvements, is determined by a VNS. This VNS is applied on the adjusted road network to construct routes for the vehicles. This adjusted network is the existing network to which the improvements in the current solution are applied. For a given set of days, routes for the vehicles are determined for each day separately. In other words, a SVRP is solved for each day in the considered group of days in the adjusted network. The applied VNS is based on the basic VNS of Hansen and Mladenovic [6]. Note that the main goal of our research is not to find the best heuristic to solve a SVRP, but to determine the best (or good) set of improvements for a road network. Therefore, we have chosen a simple and fast 


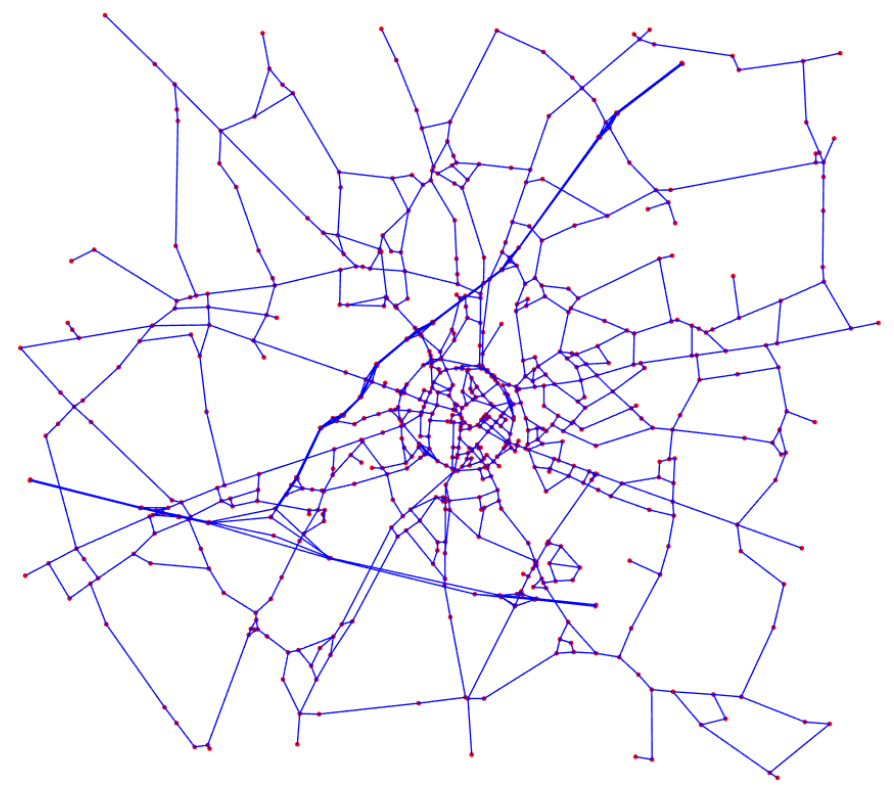

Fig. 1. Network of Leuven

algorithm to obtain a fair enough estimation of the number of vehicle kilometers in the adjusted network.

First of all, the VNS needs an initial solution to start its exploration of the solution space. This initial solution is constructed by a nearest neighbor algorithm. Secondly, a set of neighborhood structures to generate random neighbors should be selected. In this application of the VNS, a set of three different neighborhood operators is used to generate random neighbors: Or-opt, Cross-exchange and 2-opt-star. These operators are based on the operators used in [8]. Every time, one operator is selected and a random neighbor is constructed. This newly generated solution is used as the initial solution for the local search step (discussed in the next paragraph). If during the local search step a solution with less vehicle kilometers is found, the solution is registered and the loop is restarted with the first operator. Otherwise, the VNS continues with the next operator. When the last operator is selected and no improvement can be found, the loop is finished. Finally, we set a stopping condition on the number of iterations. An iteration of the VNS consists of a complete loop of the set of operators to generate random neighbors. Since this VNS is applied for each newly generated solution during the ALNS, we have decided to stop our VNS already after one iteration to obtain a fast estimation of the number of vehicle kilometers.

The Variable Neighborhood Descent (VND) is the local search step within our VNS. In the VND, a different set of neighborhood operators is considered: Exchange-intra-route, 2-opt, Relocate, Exchange-inter-route. Also these operators are based on the operators used in [8]. These operators are applied consecutively to the current solution. When the best neighbor of a specific neighborhood has less vehicle kilometers than the current solution, this neighbor solution is set as the new current solution and the same operator is applied to this solution.
TABLE I

AN OVERVIEW OF THE GENERATED BENCHMARK INSTANCES

\begin{tabular}{c|c|c|c|c}
$\begin{array}{c}\text { \# of } \\
\text { Instance }\end{array}$ & $\begin{array}{c}\text { \# of } \\
\text { Nodes }\end{array}$ & $\begin{array}{c}\text { \# of } \\
\text { Customers }\end{array}$ & $\begin{array}{c}\text { \# of } \\
\text { Vehicles }\end{array}$ & Capacity \\
\hline 1 & 485 & 98 & 1 & 40 \\
2 & 485 & 98 & 2 & 20 \\
3 & 485 & 98 & 3 & 14 \\
4 & 485 & 98 & 4 & 10 \\
\hline 5 & 485 & 98 & 1 & 75 \\
6 & 485 & 98 & 2 & 38 \\
7 & 485 & 98 & 3 & 25 \\
8 & 485 & 98 & 4 & 19 \\
\hline 9 & 721 & 185 & 1 & 70 \\
10 & 721 & 185 & 2 & 35 \\
11 & 721 & 185 & 3 & 24 \\
12 & 721 & 185 & 4 & 18 \\
\hline 13 & 721 & 185 & 1 & 136 \\
14 & 721 & 185 & 2 & 68 \\
15 & 721 & 185 & 3 & 46 \\
16 & 721 & 185 & 4 & 34
\end{tabular}

Otherwise, the next operator is applied to the current solution. This process is repeated until none of the considered operators can construct a neighbor with a lower travel distance than the current solution.

\section{EXPERIMENTAL RESUlTS}

In this section, we will show some experimental results that indicate the savings that can be obtained by improving the network. In the first subsection, a set of 16 benchmark instances is generated and the used parameters in our ALNS are discussed. In the second subsection, the obtained reduction in vehicle kilometers by improving the network is discussed.

\section{A. Benchmark Instances}

In this subsection, we will discuss the generated benchmark instances. To test the performance of the presented heuristic, the current road network in and around the city of Leuven in Belgium is used. This road network consists of 721 nodes of which 185 nodes are customer nodes. This network is displayed in Figure 1. From this network, we also generated a subnetwork around the city center. This subnetwork consists of 485 nodes of which 98 nodes are customer nodes. In the subnetwork, there are around 3400 possible improvements, while in the large network, there are around 5000 possible improvements. On each network, we have generated eight benchmark instances.

For both networks, a set of 20 days of demand is considered. In the first set of benchmark instances on each network, on each day, each customer has a probability of one third to have a demand, which is always equal to exactly one item. In the second set of benchmark instances on each network, the probability of each customer to have a demand on a certain day is equal to two thirds. Both networks and both demand patterns are considered in this research with one up to four vehicles. Consequently, we have generated 16 benchmark instances on these two road networks. An overview of the generated benchmark instances is displayed in Table I. 
TABLE II

REDUCTION IN VEHICLE KILOMETERS IN THE 16 BENCHMARK INSTANCES WITH A BUDGET OF 5 UNITS

\begin{tabular}{c||r|r|r}
\multicolumn{1}{c||}{$\begin{array}{c}\text { \# } \\
\text { of } \\
\text { Instance }\end{array}$} & $\begin{array}{c}\text { Best } \\
\text { Reduction }\end{array}$ & $\begin{array}{c}\text { Average } \\
\text { Reduction }\end{array}$ & CPU (s) \\
\hline 1 & $2.35 \%$ & $2.30 \%$ & 5716 \\
2 & $2.88 \%$ & $2.09 \%$ & 5787 \\
3 & $2.11 \%$ & $0.94 \%$ & 5732 \\
4 & $1.98 \%$ & $0.91 \%$ & 5674 \\
\hline 5 & $2.92 \%$ & $1.51 \%$ & 6828 \\
6 & $2.77 \%$ & $1.82 \%$ & 7219 \\
7 & $2.69 \%$ & $1.76 \%$ & 6390 \\
8 & $3.40 \%$ & $1.80 \%$ & 6222 \\
\hline 9 & $2.19 \%$ & $1.03 \%$ & 18086 \\
10 & $1.81 \%$ & $1.03 \%$ & 18324 \\
11 & $2.74 \%$ & $1.56 \%$ & 18011 \\
12 & $1.04 \%$ & $0.66 \%$ & 17747 \\
\hline 13 & $2.74 \%$ & $1.14 \%$ & 24803 \\
14 & $2.73 \%$ & $1.42 \%$ & 24524 \\
15 & $1.98 \%$ & $1.41 \%$ & 20627 \\
16 & $2.40 \%$ & $1.47 \%$ & 18851 \\
\hline Average & $2.42 \%$ & $1.43 \%$ & -
\end{tabular}

The total sum of the capacity of the vehicles is equal to the largest number of customers which have demand on one day. It is assumed that all vehicles have the same capacity. Furthermore, we assume that both widening an arc and converting an edge leads to a travel time reduction of $25 \%$ on this arc or edge. Furthermore, in this research, all 16 benchmark instances are considered with a total available budget of 5 units and of 10 units. The costs for converting an edge are between 1 and 2 units. For adding and edge, the costs are between 2 and 4 units, while the costs of widening an arc are between 3 and 5 units. The exact cost depends on the relative length of the edges or arcs that can be changed. Consequently, the number of improvements in a set is between 1 and 4 improvements in case of a budget of 5 units and in the case of a budget of 10 units, the number of improvements in a set is between 2 to 9 improvements.

\section{B. Parameter Setting}

The parameters which are used in our ALNS are discussed in this subsection. Our ALNS heuristic, presented in Section III, contains many parameters. Based on preliminary experiments, we concluded that the best values for these parameters are the following. For determining the start temperature of the SA structure, we used a percentage $p$ equal to $25 \%$. The cooling down parameter $\alpha$ of the temperature is, in this research, equal to 0.75 , while the temperature is adjusted every 10 iterations $(i=10)$. In every iteration, the part $d$ of the solution which is destroyed, is equal to 0.5 . In order to determine the new weights, the score $\pi_{i}$ is increased by $\sigma_{1}$ equal to $47, \sigma_{2}$ equal to 12 and $\sigma_{3}$ equal to 13 , if the newly generated solution is respectively better than the best known solution up to that far, better than the current solution or accepted by the SA but worse than the current solution. Every 10 iterations $(j=10)$, the weights of the destroy and repair methods are adapted with a reaction factor $r$ equal to 0.1 to the performance of the methods during these 10 iterations.
TABLE III

REDUCTION IN VEHICLE KILOMETERS IN THE 16 BENCHMARK INSTANCES WITH A BUDGET OF 10 UNITS

\begin{tabular}{c||r|r|r}
\multicolumn{1}{c||}{\begin{tabular}{c}
\multirow{2}{*}{$\begin{array}{c}\text { Budget } \\
\text { of } \\
\text { Instance }\end{array}$} \\
Reduction
\end{tabular}} & $\begin{array}{c}\text { Bverage } \\
\text { Reduction }\end{array}$ & CPU (s) \\
\hline 1 & $3.83 \%$ & $3.31 \%$ & 5851 \\
2 & $3.82 \%$ & $2.79 \%$ & 5938 \\
3 & $3.17 \%$ & $2.52 \%$ & 5880 \\
4 & $2.53 \%$ & $2.26 \%$ & 5818 \\
\hline 5 & $4.78 \%$ & $2.86 \%$ & 6956 \\
6 & $3.96 \%$ & $2.49 \%$ & 7334 \\
7 & $4.93 \%$ & $2.90 \%$ & 6467 \\
8 & $3.29 \%$ & $2.42 \%$ & 6260 \\
\hline 9 & $2.16 \%$ & $1.44 \%$ & 17090 \\
10 & $2.25 \%$ & $1.32 \%$ & 16955 \\
11 & $2.70 \%$ & $1.77 \%$ & 16524 \\
12 & $2.29 \%$ & $1.78 \%$ & 16225 \\
\hline 13 & $3.10 \%$ & $2.26 \%$ & 26597 \\
14 & $2.60 \%$ & $2.13 \%$ & 26004 \\
15 & $3.33 \%$ & $2.74 \%$ & 21028 \\
16 & $3.21 \%$ & $2.29 \%$ & 19273 \\
\hline Average & $3.25 \%$ & $2.33 \%$ & -
\end{tabular}

In this research, the total number of iterations is equal to 200 $(I=200)$, which means that both the temperature and the weights are adjusted 10 times during our ALNS heuristic.

\section{Reduction in vehicle kilometers by changing the network}

In both Table II and Table III, an indication of the reduction in vehicle kilometers by a set of network changes is presented. In order to obtain these results, we have applied our ALNS heuristic ten times to each of the 16 benchmark instances for both given budgets. As output of these ten runs, there are ten (different) sets of improvements. To determine the percentage reduction in vehicle kilometers of each set of improvements, the required kilometers of the vehicles during the group of days in the adjusted network is compared with the number of vehicle kilometers in the original network. Both values are determined by the presented VNS heuristic with corresponding VND. In this evaluation process, the heuristic is stopped after 100 iterations. The set of improvements with the smallest number of vehicle kilometers in the adjusted network is set to be the best set of improvements. The percentage reduction in vehicle kilometers in the network adjusted by this best set of improvements compared to the vehicle kilometers in the original network is presented in the second column of Table II and Table III.

In order to show the performance of our ALNS over the ten runs, the average percentage reduction in vehicle kilometers over the ten runs is displayed in the third column of Table II and Table III. The fourth column contains the total calculation time over the ten runs. Note that in this calculation time, only the time which is required to determine the best set of improvements by our ALNS is considered. This means that the time which is required for the evaluation process afterwards, with 100 iterations, is not considered.

Table II shows that the average reduction in vehicle kilometers is equal to $2.42 \%$ over all the 16 benchmark instances in case of a given budget of 5 units, while in Table III is 
displayed that, in case of a given budget of 10 units, the average reduction in vehicle kilometers is equal to $3.25 \%$.

Besides that, the average reduction in the subnetwork is equal to $3.21 \%$, while in the large network, the average reduction is equal to $2.46 \%$. This difference in reduction is due to the fact that in both networks, the available budget is sufficient to implement a small set of improvements. These improvements will have a larger effect on the number of vehicle kilometers in a smaller network than in a large network. In order to obtain the same reduction in vehicle kilometers in a larger network, it is necessary to have a larger budget available to implement a larger set of improvements in the network.

Moreover, in the first demand pattern, the average reduction is equal to $2.49 \%$, while the reduction in the second demand pattern is on average equal to $3.18 \%$. This is due to the fact that the number of customers that requires a visit on a day is larger in the second demand pattern than in the first demand pattern. This means that the routes of the vehicles are longer and the added edges and improved arcs can be used more often and therefore, the effect of the network changes will be larger.

Furthermore, investigating the best sets of improvements of all instances, we can determine that in these sets, only edges to (re-)open or to convert are considered. The costs of these improvements are relatively small, while the effect on the reduction of the number of vehicle kilometers is large. Besides that, based on the weights of the used destroy methods at the end of the ALNS heuristic, we can determine that both the random destroyment and the destroyment of the changes which are in the solution for the largest number of iterations have a positive effect on quality of the solution. Considering the weights of the repair methods, we can determine that adding the most beneficial improvements has a large positive effect on the quality of the solution.

\section{CONCLUSiON}

In this research, we have considered the possible reduction in logistic vehicle kilometers that can be obtained when a cooperation of delivery companies has the ability to suggest a set of network improvements to the local government. Different network changes are considered in our research: reopening existing roads for the vehicles of the cooperation, widening roads in the network or converting existing roads into a one-way road with a higher speed. To find the best set of improvements given a fixed budget in a realistic road network, a ALNS heuristic is proposed. In order to get an indication of the possible reduction in vehicle kilometers and to test the performance of the heuristic, experiments on a set of 16 benchmark instances are executed. These benchmark instances are generated from a realistic city road network. In these instances, different network sizes, different demand patterns and different numbers of vehicles are considered. All generated benchmark instances are considered with two different available budgets. Based on these experimental results, we can conclude that a set of 1 up to 4 network improvements will lead to a reduction in vehicle kilometers of on average $2.42 \%$ over these 16 benchmark instances, while implementing a set of 2 up to 9 improvements will lead to a reduction equal to $3.25 \%$. Furthermore, the reduction in vehicle kilometers is larger when the considered road network is smaller. Also, if the routes of the vehicles are longer, the reduction in vehicle kilometers, due to the network changes, will be larger.

In further research, multiple depots outside the city center could be considered. In this research, we assumed that all products that should be delivered by the cooperation are collected in one depot outside the city center. However, considering multiple depots to collect the products to deliver could be an interesting extension of the problem. Besides that, in this research, only the 'last mile' transport of the cooperation is considered, since the delivering of the products to the depot is ignored. In further research, it could be useful to investigate both the delivering to the depots and the delivering to the customers in the city area simultaneously. Finally, considering time windows for the customers in the city area and/or the roads in the network could also be an interesting path for further research.

\section{ACKNOWLEDGMENT}

This research was funded by a Ph.D. grant of the Agency for Innovation by Science and Technology in Flanders (IWT).

\section{REFERENCES}

[1] J.N. Al-Karaki and A.E. Kamal. Routing techniques in wireless sensor networks: a survey. Wireless communications, 11(6):6-28, 2004.

[2] E. Bogers, T. Langerak, H. Jordaan and S. Weijers. Haalbaarheid stadslogistiek in Zutphen: een concrete studie voor twee potentiële klanten. Logistiek: Tijdschrift voor toegepaste logistiek, 1:108-121, 2016 [In Dutch].

[3] D. Canca, A. De-Los-Santos, G. Laporte and J.A. Mesa. An adaptive neighborhood search metaheuristic for the integrated railway rapid transit network design and line planning problem. Computers \& Operations Research, 78:1-14, 2017.

[4] L. Chen and W.B. Heinzelman. A Survey of Routing Protocols that Support QoS in Mobile Ad Hoc Networks. Networks, 21(6):30-38, 2007.

[5] B. Fleischmann. A cutting plane procedure for the Travelling Salesman Problem on road networks. European Journal of Operational Research, 21(3):307-317, 1985.

[6] P. Hansen and N. Mladenovic. Variable neigborhood search: Principles and applications. European Journal of Operational Research, 130(3):449 467, 2001.

[7] G. Kant, H. Quak, R. Peeters and T. van Woensel. Urban freight transportation: challenges, failures and successes. In Logistics and Supply Chain Innovation, 127-139, 2016.

[8] J. Kytöjoki, T. Nuortio, O. Bräysy and M. Gendreau. An efficient variable neighborhood search heuristic for very large scale vehicle routing problems. Computers \& Operations Research, 34(9):2743-2757, 2007.

[9] A.N. Letchford, S.D. Nasiri and D.O. Theis. Compact formulations of the Steiner Traveling Salesman Problem and related problems. European Journal of Operational Research, 228(1):83-92, 2012.

[10] S. Ropke and D. Pisinger. An adaptive large neighborhood search heuristic for the pickup and delivery problem with time windows. Transportation science, 40(4):455-472, 2006.

[11] M. Samà, A. D'Ariano, F. Corman and D. Pacciarelli. Metaheuristics for efficient aircraft scheduling and re-routing at busy terminal control areas. Transportation Research Part C: Emerging Technologies, DOI:10.1016/j.trc.2016.08.012, 2016.

[12] M. Samà, A. D'Ariano, F. Corman and D. Pacciarelli. A variable neighbourhood search for fast train scheduling and routing during disturbed railway traffic situations. Computers \& Operations Research, 78:480-499, 2017.

[13] J.F. Sze, S. Salhi and N. Wassan. A hybridisation of adaptive variable neighbourhood search and large neighbourhood search: Application to the vehicle routing problem. Expert Systems with Applications, 65:383-397, 2016. 